\title{
Resolutions of the Coulomb operator
}

\section{Part III. $\dagger$ Reduced-rank Schrödinger equations $\ddagger$}

\section{Taweetham Limpanuparb and Peter M. W. Gill*}

Received (in CAMBS) 29th May 2009, Accepted 13th July 2009

First published as an Advance Article on the web 14th August 2009

DOI: $10.1039 / \mathrm{b} 910613 \mathrm{~h}$

We consider a modified Schrödinger equation wherein the electron-electron repulsion terms $r_{i j}{ }^{-1}$ are approximated by truncated one-particle resolutions. Numerical results for the He atom and $\mathrm{H}_{2}$ molecule at the Hartree-Fock, second-order Møller-Plesset, and configuration interaction levels show that the solutions of the resulting reduced-rank Schrödinger equations converge rapidly, and that even low-rank approximations can yield energies with chemical accuracy.

\section{Introduction}

The chief difficulty in applying quantum mechanics to problems in chemical physics is that the Coulomb operators $r_{i j}{ }^{-1} \equiv$ $\left|\boldsymbol{r}_{i}-\boldsymbol{r}_{j}\right|^{-1}$, which pervade the relevant Hamiltonians, couple the motions of the particles. It is this coupling that lies at the heart of the Coulomb problem, ${ }^{1}$ the exchange problem ${ }^{2}$ and, in particular, the notorious electron correlation problem. ${ }^{3}$

Although ongoing research efforts have produced a range of methods to address this fundamental difficulty, including separating the Coulomb operator into its short- and longrange components, ${ }^{4-8}$ treatment of the short-range component by specialized techniques ${ }^{9-11}$ and the long-range component by multipole expansion, ${ }^{12-14}$ complete neglect of the longrange component, ${ }^{9,15,16}$ and treatment of the operator in Fourier space, ${ }^{6,17,18}$ none of these has yet yielded a comprehensive solution to the correlation problem.

In the earlier papers ${ }^{19,20}$ in this series, henceforth I and II, we introduced a resolution

$$
r_{i j}^{-1}=\sum_{n l m}^{\infty} \phi_{n l m}^{*}\left(\boldsymbol{r}_{i}\right) \phi_{n l m}\left(\boldsymbol{r}_{j}\right) \equiv \sum_{k}^{\infty} \phi_{k}^{*}\left(\boldsymbol{r}_{i}\right) \phi_{k}\left(\boldsymbol{r}_{j}\right)
$$

of the two-particle Coulomb operator into one-particle potentials

$$
\phi_{n l m}(\boldsymbol{r})=2 \sqrt{2} Y_{l m}(\boldsymbol{r}) V_{n l}(r)
$$

where the radial potentials are given by

$$
V_{n l}(r)=\int_{0}^{\infty} h_{n}(x) j_{l}(x r) \mathrm{d} x
$$

and where $Y_{l m}$ is a spherical harmonic, $j_{l}$ is a spherical Bessel function $^{21}$ and the $h_{n}(x)$ are a set of functions that are complete and orthonormal on $[0, \infty)$.

This "resolution of the Coulomb operator" (RC) is analogous to the familiar "resolution of the identity" (RI) ${ }^{22-25}$ and allows

Research School of Chemistry, Australian National University, CanberraACT 0200,Australia.E-mail: peter.gill@anu.edu.au

$\dagger$ For Parts I and II see ref. 19 and 20.

$\ddagger$ Electronic supplementary information (ESI) available: Auxiliary integrals in the $R=0$ case. See DOI: $10.1039 / \mathrm{b} 910613 \mathrm{~h}$ us to expand Coulomb matrix elements into auxiliary integrals, i.e.

$$
\left(a\left|r_{12}^{-1}\right| b\right)=\sum_{n l m}^{\infty}\left(a \mid \phi_{n l m}\right)\left(\phi_{n l m} \mid b\right) \equiv \sum_{k}^{\infty}\left(a \mid \phi_{k}\right)\left(\phi_{k} \mid b\right)
$$

If the resolution is truncated after $\mathscr{K}<\infty$ terms, the resulting rank- $\mathscr{K}$ approximation

$$
r_{i j}^{-1} \approx \sum_{n=0}^{\mathscr{N}} \sum_{l=0}^{\mathscr{L}} \sum_{m=-l}^{l} \phi_{n l m}^{*}\left(\boldsymbol{r}_{i}\right) \phi_{n l m}\left(\boldsymbol{r}_{j}\right) \equiv \sum_{k=1}^{\mathscr{K}} \phi_{k}^{*}\left(\boldsymbol{r}_{i}\right) \phi_{k}\left(\boldsymbol{r}_{j}\right)
$$

which we will call the $[\mathscr{N}, \mathscr{L}]$ resolution, yields integral approximations

$$
\begin{aligned}
\left(a\left|r_{12}^{-1}\right| b\right) & \approx \sum_{n=0}^{\mathscr{N}} \sum_{l=0}^{\mathscr{L}} \sum_{m=-l}^{l}\left(a \mid \phi_{n l m}\right)\left(\phi_{n l m} \mid b\right) \\
& \equiv \sum_{k}^{\mathscr{K}}\left(a \mid \phi_{k}\right)\left(\phi_{k} \mid b\right)
\end{aligned}
$$

that are reminiscent of the Cholesky decompositions ${ }^{25-28}$ and Kronecker approximations ${ }^{29,30}$ currently being developed. We note that $\mathscr{K} \equiv(\mathscr{N}+1)(\mathscr{L}+1)^{2}$ and we choose to relate the index $k$ to the indices $n, l$ and $m$ through $k=n(\mathscr{L}+1)^{2}+$ $l(l+1)+m+1$.

Of the myriad ways to resolve the Coulomb operator, we have previously explored two. In I, we chose the generating functions $h_{n}$ to be even-order Hermite functions weighted by a Gaussian but observed that calculation of the resulting $V_{n l}(r)$ is unwieldy when $\mathscr{N}$ or $\mathscr{L}$ are large. In II, we chose

$$
h_{n}(x)=\sqrt{2} L_{n}(2 x) \exp (-x)
$$

where $L_{n}$ is a Laguerre polynomial ${ }^{21}$ and this yields a resolution with potentials such as

$$
\begin{aligned}
& \phi_{0,0,0}(r)=\frac{2}{\sqrt{\pi}}\left[\frac{\tan ^{-1} r}{r}\right] \\
& \phi_{1,0,0}(r)=\frac{2}{\sqrt{\pi}}\left[\frac{\tan ^{-1} r}{r}-\frac{2}{1+r^{2}}\right] \\
& \phi_{n, 0,0}(r)=\frac{2}{\sqrt{\pi}} \frac{(-1)^{n}}{r} \Im\left\{B_{z}(n+1,-n)\right\}
\end{aligned}
$$


where $\mathfrak{I}$ is the imaginary part, $B_{z}$ is the incomplete beta function $^{21}$ and $z=(1+i r) / 2$. These are better behaved numerically and were also adopted in recent work by Hoggan. ${ }^{31}$

In II, we studied the rate of convergence of the Coulomb self-interaction energy of the hydrogenic ions $\left(\mathrm{H}, \mathrm{He}^{+}, \mathrm{Li}^{2+}\right.$, $\mathrm{Be}^{3+}$ and $\mathrm{B}^{4+}$ ) with respect to $\mathscr{K}$ and found that the behaviour deteriorates as the nuclear charge increases. This arises because the physical size of the potentials $\phi_{k}(\boldsymbol{r})$ becomes increasingly poorly matched to the electron densities, $\rho(\boldsymbol{r})$, which shrink toward the nuclei as the nuclear charge increases. The problem can be solved simply by compressing the density (or orbitals) by a well-chosen scale factor, $\mathscr{Z}$, applying the Coulomb resolution, and then re-scaling the resulting energy by the same factor. Alternatively, of course, one could scale the $\phi_{k}(\boldsymbol{r})$ to match the density but we prefer, for aesthetic reasons, not to introduce a $\mathscr{Z}$-dependence into the potentials.

In the present paper, we explore the consequences of replacing the electron-electron terms in the molecular Schrödinger equation by their rank- $\mathscr{K}$ approximations, eqn (1.5). We discuss results for $\mathrm{He}$ and $\mathrm{H}_{2}$ at the Hartree-Fock (HF), second-order Møller-Plesset (MP2) and configuration interaction (CI) levels. Atomic units and real orbitals are used throughout.

\section{Reduced-rank Schrödinger equations}

The non-relativistic electronic Schrödinger equation for an $N$-electron system is

$$
\left[\sum_{i}^{N} \hat{h}\left(\boldsymbol{r}_{i}\right)+\sum_{i<j}^{N} r_{i j}^{-1}\right] \Psi=E \Psi
$$

where $\hat{h}$ is the one-electron operator describing an electron's kinetic energy and its interaction with an external field, such as the nuclei. Replacing the problematic electron repulsion terms by the rank- $\mathscr{K}$ approximation, eqn (5), yields the reducedrank Schrödinger equation (RRSE)

$$
\begin{aligned}
& {\left[\sum_{i}^{N} \hat{h}\left(\boldsymbol{r}_{i}\right)+\frac{1}{2} \sum_{k}^{\mathscr{K}}\left|\sum_{i}^{N} \phi_{k}\left(\boldsymbol{r}_{i}\right)\right|^{2}\right.} \\
& \left.-\frac{1}{2} \sum_{k}^{\mathscr{K}} \sum_{i}^{N}\left|\phi_{k}\left(\boldsymbol{r}_{i}\right)\right|^{2}\right] \Psi=E \Psi
\end{aligned}
$$

and, of course, as $\mathscr{K} \rightarrow \infty$, we recover the original Schrödinger equation, eqn (11).

At first glance, the RRSE may appear more complicated than the original Schrödinger equation, but this is not so. The third term in eqn (12) consists of one-electron contributions and therefore presents no difficulty. The second term is more challenging but still offers a considerable simplification over the original equation.

In II, we showed that the Laguerre resolution yields surprisingly rapid convergence of Coulomb and exchange energies and it is therefore interesting to see how well the solutions of eqn (12) mimic those of eqn (11) as $\mathscr{K}$ increases.

At points where two electrons coincide, i.e. $\boldsymbol{r}_{i}=\boldsymbol{r}_{j}$, the Hamiltonian in eqn (11) is singular and this leads to cusps in the exact wavefunction ${ }^{32}$. In contrast, for finite $\mathscr{K}$, the
Hamiltonian in eqn (12) is non-singular at such points and the exact solutions of the RRSE therefore lack such cusps. We therefore expect that the approximate solutions of the RRSE will converge more rapidly with respect to the size of the one-electron basis than those of the original Schrödinger equation. ${ }^{33,34}$

In this investigation, we will confine our attention to the special case of two-electron systems, for which the RRSE reduces to

$$
\left[\hat{h}\left(\boldsymbol{r}_{1}\right)+\hat{h}\left(\boldsymbol{r}_{2}\right)+\sum_{k}^{\mathscr{K}} \phi_{k}\left(\boldsymbol{r}_{1}\right) \phi_{k}\left(\boldsymbol{r}_{2}\right)\right] \Psi=E \Psi
$$

We anticipate that the general conclusions that emerge from this study will also apply to larger systems, and preliminary studies on Be and $\mathrm{LiH}$ confirm this.

\section{Reduced-rank quantum chemistry models}

\section{A Hartree-Fock theory}

In conventional Hartree-Fock (HF) theory for a two-electron singlet, the Fock operator is

$$
\hat{F}=\hat{h}+\hat{J}
$$

where $\hat{J}$ is the full Coulomb operator. If, however, we develop a reduced-rank Hartree-Fock (RRHF) theory based on the RRSE (eqn (13)), the associated Fock operator becomes

$$
\hat{F}^{\mathscr{K}}=\hat{h}+\hat{J}^{\mathscr{K}}
$$

and, in a finite basis of size $B$, the resulting Fock matrix elements $^{35}$ are given by

$$
F_{\mu \nu}^{\mathscr{K}}=(\mu|\hat{h}| \nu)+\sum_{k}^{\mathscr{K}}\left(\mu \nu \mid \phi_{k}\right)\left(\phi_{k} \mid \rho\right)
$$

where

$$
\left(\phi_{k} \mid \rho\right)=\sum_{\mu \nu} P_{\mu \nu}\left(\mu \nu \mid \phi_{k}\right)
$$

and $P_{\mu \nu}$ is a density matrix element. This shows that an RRHF calculation is analogous to a conventional HF one, except that $\mathcal{O}\left(B^{4}\right)$ two-electron integrals $(\mu \nu \mid \lambda \sigma)$ are replaced by $\mathcal{O}\left(B^{2} \mathscr{K}\right)$ auxiliary integrals $\left(\mu \nu \mid \phi_{k}\right)$. This is reminiscent of the RI and Cholesky schemes but, of course, there is no RRHF metric matrix to invert.

To obtain an initial guess for the self-consistent field (SCF) algorithm, we diagonalize the core Hamiltonian matrix, and to transform the Fock matrix into an orthonormal basis, we use symmetric orthonormalization. We terminate the SCF iterations when the RMS change in the density matrix falls below $10^{-4}$. Our algorithm for calculating the auxiliary integrals is discussed in section IV, below.

We define $E_{\mathrm{HF}}^{\mathcal{V}, \mathscr{L}}$ as the ground-state restricted HF energy from the $[\mathscr{N}, \mathscr{L}]$ resolution, and it is convenient to quantify its error by

$$
\Delta_{\mathrm{HF}}^{\mathcal{N}, \mathscr{L}}=-\log _{10}\left(E_{\mathrm{HF}}-E_{\mathrm{HF}}^{\mathcal{N}, \mathscr{L}}\right)
$$

There are several ways to introduce the resolution into post-HF calculations. Henceforth, we use the $[\mathscr{N}, \mathscr{L}]$ resolution 
to generate the orbitals and orbital energies and then employ the same resolution when computing any required molecular orbital (MO) integrals. In this way, we are employing the same reduced-rank Hamiltonian throughout.

\section{B Perturbation theory}

The second-order Møller-Plesset (MP2) correlation energy ${ }^{35}$ is given by

$$
E_{\mathrm{MP} 2}=\frac{1}{4} \sum_{a b r s} \frac{|\langle a b|| r s\rangle|^{2}}{\varepsilon_{a}+\varepsilon_{b}-\varepsilon_{r}-\varepsilon_{s}}
$$

where $a, b$ are occupied and $r, s$ are virtual spin orbitals. In a closed-shell two-electron system, this reduces to a sum over virtual spatial orbitals

$$
E_{\mathrm{MP} 2}=\sum_{r s} \frac{2(1 r \mid 1 s)(11 \mid r s)-(1 r \mid 1 s)^{2}}{2 \varepsilon_{1}-\varepsilon_{r}-\varepsilon_{s}}
$$

and, thus, the reduced-rank second-order Møller-Plesset (RRMP2) energy and its error are

$$
\begin{aligned}
& E_{\mathrm{MP} 2}^{\mathcal{N}, \mathscr{L}}= \\
& \sum_{r s} \frac{2\left[\sum_{k}^{\mathscr{K}}\left(1 r \mid \phi_{k}\right)\left(\phi_{k} \mid 1 s\right)\right]\left[\sum_{k}^{\mathscr{K}}\left(11 \mid \phi_{k}\right)\left(\phi_{k} \mid r s\right)\right]-\left[\sum_{k}^{\mathscr{K}}\left(1 r \mid \phi_{k}\right)\left(\phi_{k} \mid 1 s\right)\right]^{2}}{2 \varepsilon_{1}-\varepsilon_{r}-\varepsilon_{s}}
\end{aligned}
$$

$\Delta_{\mathrm{MP} 2}^{\mathscr{N}, \mathscr{L}}=-\log _{10}\left(E_{\mathrm{MP} 2}^{\mathcal{N}, \mathscr{L}}-E_{\mathrm{MP} 2}\right)$

\section{Configuration interaction}

The full configuration interaction (FCI) correlation energy, $E_{\mathrm{FCI}}$, is the lowest eigenvalue of the blocked full CI matrix ${ }^{35}$

$$
\mathbf{H}=\left(\begin{array}{ccc}
\left\langle\Psi_{0}\left|\hat{H}-E_{\mathrm{HF}}\right| \Psi_{0}\right\rangle & 0 & \left\langle D|\hat{H}| \Psi_{0}\right\rangle \\
0 & \left\langle S\left|\hat{H}-E_{\mathrm{HF}}\right| S\right\rangle & \langle D|\hat{H}| S\rangle \\
\left\langle\Psi_{0}|\hat{H}| D\right\rangle & \langle S|\hat{H}| D\rangle & \left\langle D\left|\hat{H}-E_{\mathrm{HF}}\right| D\right\rangle
\end{array}\right)
$$

where the Hamiltonian $\hat{H}$ is defined in eqn (11) and $\Psi_{0}, S$ and $D$ are the ground-state, singly-substituted, and (spin-adapted) doubly-substituted determinants, respectively. The largest block is $\left\langle D\left|\hat{H}-E_{\mathrm{HF}}\right| D\right\rangle$ and, when $r, s, t$ and $u$ are all distinct, the CI matrix element is

$$
\left\langle\Psi_{11}^{r s}\left|\hat{H}-E_{\mathrm{HF}}\right| \Psi_{11}^{t u}\right\rangle=(r t \mid s u)+(r u \mid t s)
$$

Using the $[\mathscr{N}, \mathscr{L}]$ resolution, this becomes

$$
\left\langle\Psi_{11}^{r s}\left|\hat{H}-E_{\mathrm{HF}}\right| \Psi_{11}^{t u}\right\rangle^{\mathscr{N}, \mathscr{L}}=\sum_{k}^{\mathscr{K}}\left[\left(r t \mid \phi_{k}\right)\left(\phi_{k} \mid s u\right)+\left(r u \mid \phi_{k}\right)\left(\phi_{k} \mid t s\right)\right]
$$

and, with each matrix element approximated likewise, the lowest eigenvalue becomes $E_{\mathrm{FCI}}^{\mathcal{S}, \mathscr{L}}$. It is convenient to quantify its error by the signed quantity

$$
\Delta_{\mathrm{FCI}}^{\mathscr{N}, \mathscr{L}}=-\operatorname{sgn}\left(E_{\mathrm{FCI}}^{\mathscr{N}, \mathscr{L}}-E_{\mathrm{FCI}}\right) \log _{10}\left|E_{\mathrm{FCI}}^{\mathscr{N}, \mathscr{L}}-E_{\mathrm{FCI}}\right|
$$

\section{Auxiliary integrals}

The calculation of auxiliary integrals $\left(\mu \nu \mid \phi_{n l m}\right)$ is central to the application of RC theory to any quantum chemical method. The RC can be used with any type of basis function and Hoggan has demonstrated ${ }^{31}$ that it works well with Slater-type functions, but we will employ Cartesian Gaussians in the present work. Boys differentiation ${ }^{36}$ can be used to derive formulae for integrals of higher angular momentum and we can therefore focus on the fundamental auxiliary integrals of the form

$$
\left(s s \mid \phi_{n l m}\right)=\int \mathrm{e}^{-\zeta_{A}|\boldsymbol{r}-\boldsymbol{A}|^{2}} \mathrm{e}^{-\zeta_{B}|\boldsymbol{r}-\boldsymbol{B}|^{2}} \phi_{n l m}(\boldsymbol{r}) \mathrm{d} \boldsymbol{r}
$$

Using the Gaussian product rule, ${ }^{35}$ this becomes

$$
\left(s s \mid \phi_{n l m}\right)=G_{A B} \int \mathrm{e}^{-\gamma^{2}|\boldsymbol{r}-\boldsymbol{R}|^{2}} \phi_{n l m}(\boldsymbol{r}) \mathrm{d} \boldsymbol{r}
$$

where $\gamma^{2}=\zeta_{A}+\zeta_{B}$ and

$$
\begin{aligned}
\boldsymbol{R} & =\left(\zeta_{A} \boldsymbol{A}+\zeta_{B} \boldsymbol{B}\right) / \gamma^{2} \\
G_{A B} & =\exp \left(-\zeta_{A} \zeta_{B}|\boldsymbol{A}-\boldsymbol{B}|^{2} / \gamma^{2}\right)
\end{aligned}
$$

Invoking Parseval's theorem and choosing the Laguerre generator, eqn (7), then yields

$$
\begin{aligned}
\left(s s \mid \phi_{n l m}\right) & =\left(2 \pi / \gamma^{2}\right)^{3 / 2} G_{A B} Y_{l m}(\boldsymbol{R}) \int_{0}^{\infty} h_{n}(x) j_{l}(R x) \mathrm{e}^{-x^{2} / 4 \gamma^{2}} \mathrm{~d} x \\
& =4\left(\pi / \gamma^{2}\right)^{3 / 2} G_{A B} Y_{l m}(\boldsymbol{R}) A_{n l}(R, \gamma)
\end{aligned}
$$

The spherical harmonics $Y_{I m}(\boldsymbol{R})$ can be computed efficiently using Libbrecht's method ${ }^{37}$ but the accurate and efficient evaluation of the radial integrals

$$
A_{n l}(R, \gamma)=\int_{0}^{\infty} L_{n}(2 x) j_{l}(R x) \exp \left[-x-\frac{x^{2}}{4 \gamma^{2}}\right] \mathrm{d} x
$$

for $n=0,1, \ldots, \mathscr{N}$ and $l=0,1, \ldots, \mathscr{L}$ is non-trivial. We show in Appendix A that they can be computed recursively from Hermite functions ${ }^{21}$ and one special function. Formulae for auxiliary integrals in the $R=0$ case are available in the ESI†.

\section{Numerical results}

\section{A Basis sets}

For the purposes of this preliminary study, we have used eventempered (ET) Gaussian basis sets ${ }^{38}$ with exponents $\zeta_{k l}=\alpha_{l} \beta_{l}^{k}$, where $k=1,2, \ldots$. The parameters $\alpha_{l}$ and $\beta_{l}$, along with the $\mathrm{HF}, \mathrm{MP} 2$ and FCI energies that they yield for the He atom and $\mathrm{H}_{2}$ molecule, are listed in Table 1. The energies are close to their respective complete basis set limits.

\section{B He atom and $\mathrm{H}_{2}$ molecule}

Table 2 shows that $E_{\mathrm{HF}}^{\mathcal{N}, \mathscr{L}}, E_{\mathrm{MP} 2}^{\mathcal{N}, \mathscr{L}}$ and $E_{\mathrm{FCI}}^{\mathcal{N}, \mathscr{L}}$ converge more or less exponentially with $\mathscr{N}$, but that the scaling factor $\mathscr{Z}$ strongly influences the convergence rate. At $\mathscr{Z}=1$, 
Table 1 Basis sets and energies of $\mathrm{He}$ and $\mathrm{H}_{2}\left(R_{\mathrm{H}-\mathrm{H}}=1.40\right)$

\begin{tabular}{lll}
\hline & $\mathrm{He}$ & $\mathrm{H}_{2}$ \\
\hline Present basis & {$[10 s 3 p 2 d]$} & {$[6 s 3 p]$} \\
Size $(B)$ & 31 & 30 \\
$\alpha_{\mathrm{s}}$ & 0.0581959 & 0.0378667 \\
$\beta_{\mathrm{s}}$ & 2.7557809 & 3.3676258 \\
$\alpha_{\mathrm{p}}$ & 0.1771338 & 0.0629787 \\
$\beta_{\mathrm{p}}$ & 3.2087624 & 3.2178374 \\
$\alpha_{\mathrm{d}}$ & 0.3458537 & \\
$\beta_{\mathrm{d}}$ & 3.5196112 & -1.133287175 \\
$E_{\mathrm{HF}}$ & -2.861647460 & -0.030496094 \\
$E_{\mathrm{MP} 2}$ & -0.035127427 & -0.038527089 \\
$E_{\mathrm{FCI}}$ & -0.040734987 & \\
& & $-1.133629572^{d}$ \\
Infinite basis & & $-0.03427^{e}$ \\
$E_{\mathrm{HF}}$ & $-2.861679996^{a}$ & $-0.04084520^{f}$ \\
$E_{\mathrm{MP} 2}$ & $-0.03740^{b}$ & \\
$E_{\mathrm{FCI}}$ & $-0.042044381^{c}$ &
\end{tabular}

${ }^{a}$ Ref. 39. ${ }^{b}$ Ref. 40. Slightly different values are also reported in ref. 41 and 42. ${ }^{c}$ Ref. 39 and 43. ${ }^{d}$ Ref. $44 .{ }^{e} R_{\mathrm{H}-\mathrm{H}}=1.40108$, ref. $42 .{ }^{f}$ Ref. 44 and 45.

microhartree accuracy is achieved at $\mathscr{N}=10$ for all three methods and we have adopted $\mathscr{Z}=1$ henceforth.

Table 3 explores the convergence of $E_{\mathrm{HF}}^{\mathcal{N}, \mathscr{L}}, E_{\mathrm{MP} 2}^{\mathcal{N}, \mathscr{L}}$ and $E_{\text {FCI }}^{\mathscr{N}, \mathscr{L}}$ with respect to $\mathscr{N}$ and $\mathscr{L}$, using dots to indicate that higher $\mathscr{L}$ provides no further improvement. Because the occupied orbital is spherical and the basis contains only $s, p$ and $d$ functions, the $\phi_{n l m}$ with $l>0, l>2$ and $l>4$ contribute nothing to the HF, MP2 and CI energies, respectively.

The MP2 correlation energies converge smoothly toward their limiting values as $\mathscr{N}$ and $\mathscr{L}$ increase but the FCI results are more interesting. At $\mathscr{L}=0$, only radial correlation energy is recovered from the basis set and, for example, $E_{\mathrm{FCI}}^{12,0}=-0.017344$ is comparable to the value -0.017349 of Goldman. ${ }^{46}$ At $\mathscr{L}=2$ and $\mathscr{L}=3$, some of the $E_{\mathrm{FCI}}^{\mathcal{N}, \mathscr{L}}$ energies are lower than the limiting value because the contributions from the $d$ functions are treated incompletely. For example, at $\mathscr{L}=2,(s d \mid s d)$ integrals are treated but $(d d \mid d d)$ are not. We conclude from this that, in practical calculations, one should ensure that $\mathscr{L} \geq 2 L$, where $L$ is the maximum angular momentum in the orbital basis set. This is consistent with comparable recommendations for $\mathrm{RI}^{47}$ and Cholesky ${ }^{48}$ calculations.

The results in Table 4 for the $\mathrm{H}_{2}$ molecule were obtained with the nuclei at $(0,0, \pm 0.70)$. Convergence is similar to that for the He atom and, although $\mathscr{N}=\mathscr{L}=\infty$ is required to achieve formal convergence, the 10,12 resolution consistently yields microhartree accuracy.

Table 2 Energy errors for He atom for various $\mathscr{N}$ and $\mathscr{Z}$ with $\mathscr{L}=\infty$

\begin{tabular}{|c|c|c|c|c|c|c|c|c|c|c|c|c|c|c|c|c|c|c|c|c|c|}
\hline \multirow[b]{2}{*}{$\mathscr{Z}=$} & \multicolumn{7}{|c|}{$\Delta_{\mathrm{HF}}^{\mathcal{N}, \mathscr{L}}$} & \multicolumn{7}{|c|}{$\Delta_{\mathrm{MP} 2}^{\mathcal{H}, \mathscr{\varphi}}$} & \multicolumn{7}{|c|}{$\Delta_{\mathrm{FCI}}^{\mathscr{S} \mathscr{L}}$} \\
\hline & $1 / 2$ & 1 & 2 & 3 & 4 & 5 & 10 & $1 / 2$ & 1 & 2 & 3 & 4 & 5 & 10 & $1 / 2$ & 1 & 2 & 3 & 4 & 5 & 10 \\
\hline $\mathscr{N}=0$ & 0.2 & 0.7 & 1.9 & 1.5 & 1.0 & 0.8 & 0.4 & 1.5 & 1.5 & 1.5 & 1.6 & 1.7 & 1.8 & 1.7 & 1.4 & 1.4 & 1.5 & 1.6 & 1.7 & 1.7 & 1.6 \\
\hline $\mathscr{N}=2$ & 1.3 & 2.8 & 2.5 & 2.2 & 2.2 & 2.2 & 1.1 & 1.6 & 2.5 & 2.8 & 2.4 & 2.1 & 2.0 & 1.8 & 1.6 & 2.7 & 2.9 & 2.4 & 2.1 & 1.9 & 1.8 \\
\hline $\mathscr{N}=4$ & 2.2 & 4.0 & 4.0 & 2.8 & 2.4 & 2.3 & 1.9 & 2.2 & 3.7 & 3.4 & 3.0 & 2.7 & 2.5 & 1.9 & 2.3 & 4.0 & 3.5 & 3.0 & 2.8 & 2.5 & 1.9 \\
\hline $\mathscr{N}=6$ & 2.9 & 4.8 & 4.7 & 3.7 & 2.9 & 2.5 & 2.3 & 2.8 & 4.6 & 4.1 & 3.4 & 3.0 & 2.9 & 2.1 & 2.9 & 4.8 & 4.3 & 3.4 & 3.1 & 2.9 & 2.1 \\
\hline $\mathscr{N}=8$ & 3.5 & 5.4 & 5.0 & 4.7 & 3.6 & 3.0 & 2.3 & 3.4 & 5.3 & 4.7 & 3.9 & 3.4 & 3.1 & 2.4 & 3.6 & 5.5 & 5.0 & 4.0 & 3.4 & 3.2 & 2.4 \\
\hline $\mathscr{N}=10$ & 4.0 & 6.0 & 5.7 & 4.8 & 4.4 & 3.5 & 2.3 & 4.0 & 5.8 & 5.2 & 4.3 & 3.7 & 3.3 & 2.6 & 4.2 & 6.0 & 5.4 & 4.5 & 3.8 & 3.4 & 2.7 \\
\hline $\mathscr{N}=12$ & 4.5 & 6.5 & 6.7 & 5.0 & 4.8 & 4.2 & 2.5 & 4.5 & 6.2 & 5.6 & 4.7 & 4.0 & 3.6 & 2.8 & 4.6 & 6.4 & 5.8 & 5.0 & 4.2 & 3.7 & 2.9 \\
\hline
\end{tabular}

Table 3 Energy errors for He atom for various $\mathscr{N}$ and $\mathscr{L}$ with $\mathscr{Z}=1$

\begin{tabular}{|c|c|c|c|c|c|c|c|c|c|c|c|c|c|c|c|}
\hline \multirow[b]{2}{*}{$\mathscr{L}=$} & \multicolumn{5}{|c|}{$\Delta_{\mathrm{HF}}^{\mathcal{H}, \mathscr{L}}$} & \multicolumn{5}{|c|}{$\Delta_{\mathrm{MP} 2}^{\mathcal{H} \mathscr{\mathscr { L }}}$} & \multicolumn{5}{|c|}{$\Delta_{\mathrm{FCI}}^{\mathscr{N} \mathscr{L}}$} \\
\hline & 0 & 1 & 2 & 3 & 4 & 0 & 1 & 2 & 3 & 4 & 0 & 1 & 2 & 3 & 4 \\
\hline $\mathscr{N}=0$ & 0.7 & . & . & . & . & 1.5 & 1.5 & 1.5 & . & . & 1.4 & 1.4 & +1.4 & +1.4 & 1.4 \\
\hline $\mathscr{N}=2$ & 2.8 & . & . & . & . & 1.6 & 2.3 & 2.5 & . & . & 1.6 & 2.5 & +2.7 & +2.7 & 2.7 \\
\hline $\mathscr{N}=4$ & 4.0 & . & . & . & . & 1.7 & 2.5 & 3.7 & . & . & 1.6 & 2.9 & -3.8 & +4.1 & 4.0 \\
\hline $\mathscr{N}=6$ & 4.8 & . & . & . & . & 1.7 & 2.5 & 4.6 & . & . & 1.6 & 2.9 & -3.6 & -5.3 & 4.8 \\
\hline $\mathscr{N}=8$ & 5.4 & . & . & . & . & 1.7 & 2.5 & 5.3 & . & . & 1.6 & 2.9 & -3.6 & -4.8 & 5.5 \\
\hline $\mathscr{N}=10$ & 6.0 & . & . & . & . & 1.7 & 2.5 & 5.8 & . & . & 1.6 & 2.9 & -3.6 & -4.7 & 6.0 \\
\hline $\mathscr{N}=12$ & 6.5 & . & . & . & . & 1.7 & 2.5 & 6.2 & . & . & 1.6 & 2.9 & -3.6 & -4.7 & 6.4 \\
\hline
\end{tabular}

Table 4 Energy errors for $\mathrm{H}_{2}$ for various $\mathscr{N}$ and $\mathscr{L}$ with $\mathscr{Z}=1$

\begin{tabular}{|c|c|c|c|c|c|c|c|c|c|c|c|c|c|c|c|c|c|c|c|c|c|}
\hline \multirow[b]{2}{*}{$\mathscr{L}=$} & \multicolumn{7}{|c|}{$\Delta_{\mathrm{HF}}^{\mathscr{H}, \mathscr{L}}$} & \multicolumn{7}{|c|}{$\Delta_{\mathrm{M} P 2}^{\mathcal{H}, \mathscr{L}}$} & \multicolumn{7}{|c|}{$\Delta_{\mathrm{FCI}}^{\mathscr{S}, \mathscr{L}}$} \\
\hline & 0 & 2 & 4 & 6 & 8 & 10 & 12 & 0 & 2 & 4 & 6 & 8 & 10 & 12 & 0 & 2 & 4 & 6 & 8 & 10 & 12 \\
\hline $\mathscr{N}=0$ & 1.4 & 1.5 & 1.5 & 1.5 & 1.5 & 1.5 & 1.5 & 1.5 & 1.6 & 1.6 & 1.6 & 1.6 & 1.6 & 1.6 & 1.4 & 1.5 & 1.5 & 1.5 & 1.5 & 1.5 & 1.5 \\
\hline $\mathscr{N}=2$ & 2.4 & 3.5 & 3.6 & 3.6 & 3.6 & 3.6 & 3.6 & 1.6 & 2.5 & 2.7 & 2.7 & 2.7 & 2.7 & 2.7 & 1.5 & 2.7 & 2.8 & 2.8 & 2.8 & 2.8 & 2.8 \\
\hline $\mathscr{N}=4$ & 2.4 & 3.9 & 4.4 & 4.4 & 4.4 & 4.4 & 4.4 & 1.6 & 2.8 & 3.7 & 3.8 & 3.8 & 3.8 & 3.8 & 1.5 & 3.1 & 4.1 & 4.2 & 4.2 & 4.2 & 4.2 \\
\hline $\mathscr{N}=6$ & 2.4 & 4.1 & 5.0 & 5.2 & 5.2 & 5.2 & 5.2 & 1.6 & 2.9 & 4.1 & 4.5 & 4.5 & 4.5 & 4.5 & 1.5 & 3.1 & 4.6 & 4.9 & 4.9 & 4.9 & 4.9 \\
\hline $\mathscr{N}=8$ & 2.4 & 4.1 & 5.3 & 6.1 & 6.5 & 6.6 & 6.7 & 1.6 & 2.9 & 4.2 & 5.0 & 5.2 & 5.2 & 5.2 & 1.5 & 3.1 & 4.8 & 5.6 & 5.8 & 5.8 & 5.8 \\
\hline $\mathscr{N}=10$ & 2.4 & 4.1 & 5.3 & 6.2 & 6.7 & 6.9 & 7.0 & 1.6 & 2.9 & 4.2 & 5.3 & 5.7 & 5.8 & 5.9 & 1.5 & 3.1 & 4.8 & 5.9 & 6.4 & 6.7 & 6.9 \\
\hline $\mathscr{N}=12$ & 2.4 & 4.1 & 5.3 & 6.2 & 6.8 & 7.3 & 7.5 & 1.6 & 2.9 & 4.3 & 5.4 & 6.0 & 6.2 & 6.4 & 1.5 & 3.1 & 4.8 & 5.9 & 6.5 & 6.9 & 7.2 \\
\hline
\end{tabular}


Table 5 Minimum $\mathscr{N}$ and $\mathscr{L}$ required to achieve milli- and microhartree accuracy $(\mathscr{Z}=1)$

\begin{tabular}{|c|c|c|c|c|c|c|c|c|c|c|c|c|c|c|c|c|c|c|c|}
\hline & \multirow[b]{2}{*}{$B^{2}$} & \multicolumn{3}{|c|}{$\Delta_{\mathrm{HF}}^{\mathscr{N}, \mathscr{L}}=3$} & \multicolumn{3}{|c|}{$\Delta_{\mathrm{HF}}^{\mathcal{N}, \mathscr{L}}=6$} & \multicolumn{3}{|c|}{$\Delta_{\mathrm{MP} 2}^{\mathcal{N}, \mathscr{Q}}=3$} & \multicolumn{3}{|c|}{$\Delta_{\mathrm{MP} 2}^{\mathcal{H}, \mathscr{P}}=6$} & \multicolumn{3}{|c|}{$\Delta_{\mathrm{FCI}}^{\mathscr{N}, \mathscr{L}}=3$} & \multicolumn{3}{|c|}{$\Delta_{\mathrm{FCI}}^{\mathcal{Y}, \mathscr{L}}=6$} \\
\hline & & $\mathscr{N}$ & $\mathscr{L}$ & $\mathscr{K}$ & $\mathscr{N}$ & $\mathscr{L}$ & $\mathscr{K}$ & $\mathscr{N}$ & $\mathscr{L}$ & $\mathscr{K}$ & $\mathscr{N}$ & $\mathscr{L}$ & $\mathscr{K}$ & $\mathscr{N}$ & $\mathscr{L}$ & $\mathscr{K}$ & $\mathscr{N}$ & $\mathscr{L}$ & $\mathscr{K}$ \\
\hline $\mathrm{He}$ & 961 & 4 & 0 & 5 & 10 & 0 & 11 & 4 & 2 & 45 & 12 & 2 & 117 & 4 & 4 & 125 & 10 & 4 & 275 \\
\hline $\mathrm{H}_{2}$ & 900 & 2 & 2 & 27 & 8 & 6 & 441 & 4 & 4 & 125 & 12 & 8 & 1053 & 4 & 2 & 45 & 10 & 8 & 891 \\
\hline
\end{tabular}

\section{Cost and accuracy}

The obvious advantage of the RC, like the RI and Cholesky schemes, is that the $\mathcal{O}\left(B^{4}\right)$ four-centre $(\mu \nu \mid \lambda \sigma)$ integrals are replaced by the $\mathcal{O}\left(B^{2} \mathscr{K}\right)$ three-centre $\left(\mu \nu \mid \phi_{n l m}\right)$ integrals. If $\mathscr{K}<\mathcal{O}\left(B^{2}\right)$ in large systems, this is clearly beneficial and Table 5 summarizes the data in Tables 3 and 4 by listing the minimum $\mathscr{N}$ and $\mathscr{L}$ required to obtain milli- and microhartree accuracy for $\mathrm{He}$ and $\mathrm{H}_{2}$. Even in these tiny systems, $\mathscr{K}$ is competitive with $B^{2}$.

The convergence with $\mathscr{N}$ and $\mathscr{L}$ is impressive. The demand on $\mathscr{L}$ stems from the fact that the orbital basis functions have angular momentum and are not concentric with $\phi_{n l m}$. However, in the cases studied here, it was easy to saturate the $\mathscr{L}$ dimension.

In II, we showed that the reduced-rank Coulomb and exchange energies in a fixed system are sums of squares and thus converge monotonically with respect to $\mathscr{K}$. Here, the convergence with $\mathscr{N}$ is more or less monotonic for the same reason. In larger systems, monotonicity may be lost because of differential Coulomb and exchange effects and the fact that the electron density changes every SCF cycle.

\section{Concluding remarks}

We have introduced a systematic hierarchy of approximations to the Schrödinger equation (SE) in which the two-electron Coulomb operator is replaced by truncated one-electron expansions. The resulting reduced-rank Schrödinger equation (RRSE) is a mathematically simpler object than the SE but reduced-rank HF, MP2 and FCI calculations on the He atom and $\mathrm{H}_{2}$ molecule reveal that the solutions of the RRSE converge rapidly towards the corresponding solutions of the SE.

In principle, any computational method that involves Coulomb operators will benefit from the $\mathrm{RC}$ technique. In particular, the Coulomb self-interaction term, $E_{\mathrm{J}}$, common to all DFT methods is especially well suited to the RC approximation. The RC technique is related to $\mathrm{RI}$ and Cholesky schemes, and future comparisons (including timing and convergence rate studies) will be important.

These preliminary investigations suggest that the RRSE may offer a potent new route to accurate calculations. We are implementing the RC technique for these and other methods in the Q-CHEM package and will report results for larger chemical systems elsewhere.

\section{Appendix A: construction of radial integrals}

Substituting the explicit formula ${ }^{21}$ for the Laguerre polynomials

$$
L_{n}(2 x)=\sum_{k=0}^{n}(-1)^{k}\left(\begin{array}{l}
n \\
k
\end{array}\right) \frac{(2 x)^{k}}{k !}
$$

into eqn (32) allows us to write the set of radial integrals as the binomial transform ${ }^{49,50}$

$$
A_{n l}=\sum_{k=0}^{n}(-1)^{k}\left(\begin{array}{l}
n \\
k
\end{array}\right) M_{k l}
$$

of the set of monomial integrals

$$
M_{k l}(R, \gamma)=\int_{0}^{\infty} \frac{(2 x)^{k}}{k !} j_{l}(R x) \exp \left[-x-\frac{x^{2}}{4 \gamma^{2}}\right] \mathrm{d} x
$$

Our algorithm first forms $M_{k l}$ boundary values, then binomially transforms these into $A_{n l}$ boundary values, and finally uses a recurrence relation (RR) to build the remaining $A_{n l}$.

Substituting Gegenbauer's integral representation ${ }^{21}$ of $j_{l}(R x)$ into eqn (A3) and integrating over $x$ yields

$$
M_{k l}(R, \gamma)=\frac{(4 \gamma)^{k}}{i^{l+1} R} \int_{\bar{Z}}^{Z} H_{-(k+1)}(u) P_{l}\left(\frac{\gamma-u}{i \gamma R}\right) \mathrm{d} u
$$

where $Z=\gamma(1+i R), H_{-k}$ is a Hermite function and $P_{l}$ is a Legendre polynomial. ${ }^{21}$ From eqn (A4), it is easy to derive the outermost boundary values (the unfilled circles in Fig. 1)

$$
\begin{aligned}
M_{-1, l} & =\delta_{l, 0} / 2 \\
M_{k, 0} & =-\frac{(4 \gamma)^{k}}{k R} \Im\left\{H_{-k}(Z)\right\}
\end{aligned}
$$

The $M_{k, 0}$ with $k>0$ can be generated stably and efficiently by backward recursion, and the $k=0$ case

$$
M_{0,0}=\frac{2}{R} \Im\left\{\int_{0}^{Z} H_{-1}(u) \mathrm{d} u\right\}
$$

can be computed, for small $|Z|$, using the Taylor series

$$
M_{0,0}=-\frac{\sqrt{\pi}}{R} \Im\left\{\sum_{j=1}^{\infty} \frac{(-Z)^{j}}{j \Gamma\left(\frac{j+1}{2}\right)}\right\}
$$
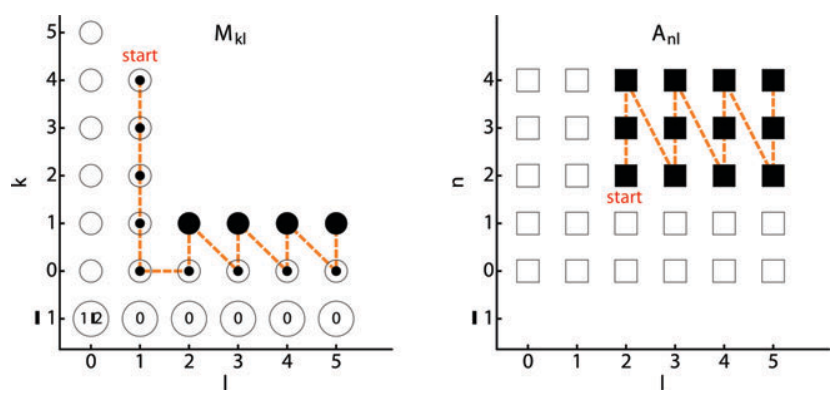

Fig. 1 A recursive pathway to generate the $M_{k l}$ and $A_{n l}$ integrals. 
and, for large $|Z|$, using the asymptotic expansion

$$
M_{0,0} \sim \frac{1}{R} \Im\left\{\ln Z-\sum_{j=1}^{\infty} \frac{\Gamma\left(j+\frac{1}{2}\right)}{2 j \sqrt{\pi}}\left(-Z^{-2}\right)^{j}\right\}
$$

Using the standard Hermite and Legendre RRs, one can derive the 5-term RR

$$
\begin{aligned}
\frac{k+1}{4 \gamma^{2}} M_{k+1, l}= & \frac{R}{2 l+1}\left[l M_{k, l-1}-(l+1) M_{k, l+1}\right] \\
& +2 M_{k-1, l}-M_{k, l}
\end{aligned}
$$

from eqn (A3), and this is used to form the $M_{k, 1}$ (the vertical dotted circles in Fig. 1). The standard Bessel RR immediately yields the 3-term RR

$$
M_{k, l}=\frac{R}{2} \frac{k+1}{2 l+1}\left(M_{k+1, l+1}+M_{k+1, l-1}\right)
$$

from eqn (A3), and this, alternating with (A10), is used to form the $M_{1, l}$ (the filled circles) and the $M_{0, l}$ (the horizontal dotted circles). We now have two rows and two columns of $M_{k l}$ values and these are binomially transformed via eqn (A2) into the corresponding rows and columns of $A_{n l}$ values (the unfilled squares). Finally, multiplying the standard Laguerre RR by the Bessel RR, one finds, from eqn (32), the 7-term RR

$$
\begin{aligned}
\frac{2(2 l+1)}{R} A_{n, l}= & (2 n+1)\left(A_{n, l+1}+A_{n, l-1}\right) \\
& -n\left(A_{n-1, l+1}+A_{n-1, l-1}\right) \\
& -(n+1)\left(A_{n+1, l+1}+A_{n+1, l-1}\right)
\end{aligned}
$$

which is used to generate all the remaining $A_{n l}$ (the filled squares in Fig. 1).

\section{Acknowledgements}

We are grateful to Dr Andrew Gilbert for useful advice in the early stages of this work and we thank the Development and Promotion of Science and Technology Talents Project for a Royal Thai Government PhD scholarship.

\section{References}

1 R. Dovesi, C. Pisani, C. Roetti and V. R. Saunders, Phys. Rev. B. Condens. Matter Mater. Phys., 1983, 28, 5781-5792.

2 E. Schwegler and M. Challacombe, J. Chem. Phys., 1996, 105, 2726-2734.

3 P.-O. Löwdin, Int. J. Quantum Chem., 1995, 55, 77-102.

4 P. P. Ewald, Ann. Phys. (Leipzig), 1921, 64, 253.

5 A. Savin, Recent Developments of Modern Density Functional Theory, Elsevier, Amsterdam, 1996.

6 J. P. Dombroski, S. W. Taylor and P. M. W. Gill, J. Phys. Chem., 1996, 100, 6272-6276.

7 A. M. Lee, S. W. Taylor, J. P. Dombroski and P. M. W. Gill, Phys. Rev. A: At., Mol., Opt. Phys., 1997, 55, 3233-3235.

8 H. Iikura, T. Tsuneda, T. Yanai and K. Hirao, J. Chem. Phys., 2001, 115, 3540-3544.

9 P. M. W. Gill and R. D. Adamson, Chem. Phys. Lett., 1996, 261, $105-110$.
10 R. D. Adamson, J. P. Dombroski and P. M. W. Gill, J. Comput. Chem., 1999, 20, 921-927.

11 S. A. Varganov, A. T. B. Gilbert and P. M. W. Gill, J. Chem. Phys., 2008, 128, 241101.

12 L. Greengard, The Rapid Evaluation of Potential Fields in Particle Systems, MIT Press, Cambridge, 1987.

13 C. A. White, B. G. Johnson, P. M. W. Gill and M. Head-Gordon, Chem. Phys. Lett., 1994, 230, 8-16.

14 M. C. Strain, G. E. Scuseria and M. J. Frisch, Science, 1996, 271, $51-53$.

15 R. D. Adamson, J. P. Dombroski and P. M. W. Gill, Chem. Phys. Lett., 1996, 254, 329-336.

16 J. Heyd, G. E. Scuseria and M. Ernzerhof, J. Chem. Phys., 2003, 118, 8207-8215.

17 G. Lippert, J. Hutter and M. Parrinello, Mol. Phys., 1997, 92, 477-487.

18 L. Fusti-Molnar and P. Pulay, J. Chem. Phys., 2002, 117, 7827-7835.

19 S. A. Varganov, A. T. B. Gilbert, E. Deplazes and P. M. W. Gill, J. Chem. Phys., 2008, 128, $201104-201107$.

20 P. M. W. Gill and A. T. B. Gilbert, Chem. Phys., 2009, 356, 86-90.

21 M. Abramowitz and I. E. Stegun, Handbook of Mathematical Functions, Dover, New York, 1972.

22 E. J. Baerends, D. E. Ellis and P. Ros, Chem. Phys., 1973, 2, $41-51$.

23 B. I. Dunlap, J. W. D. Connolly and J. R. Sabin, J. Chem. Phys., 1979, 71, 3396-3402.

24 O. Vahtras, J. Almlöf and M. Feyereisen, Chem. Phys. Lett., 1993, 213, 514-518.

25 F. Weigend, M. Kattannek and R. Ahlrichs, J. Chem. Phys., 2009, 130, 164106-164114.

26 N. H. F. Beebe and J. Linderberg, Int. J. Quantum Chem., 1977, 12, 683-705.

27 F. Aquilante, R. Lindh and T. B. Pedersen, J. Chem. Phys., 2007, 127, 114107-114113.

28 F. Aquilante, L. Gagliardi, T. B. Pedersen and R. Lindh, J. Chem. Phys., 2009, 130, 154107-154115.

29 W. Hackbusch and B. N. Khoromskij, Computing, 2006, 76, 177-202.

30 S. R. Chinnamsetty, M. Espig, B. N. Khoromskij, W. Hackbusch and H.-J. Flad, J. Chem. Phys., 2007, 127, 084110-084123.

31 P. E. Hoggan, Int. J. Quantum Chem., 2009, 109, 2926-2932.

32 T. Kato, Comm. Pure Appl. Math., 1957, 10, 151-177.

33 C. Schwartz, Phys. Rev., 1962, 126, 1015-1019.

34 W. Kutzelnigg and J. D. Morgan III, J. Chem. Phys., 1992, 96, 4484-4508.

35 A. Szabo and N. S. Ostlund, Modern Quantum Chemistry, McGraw-Hill, New York, 1989.

36 S. F. Boys, Proc. Roy. Soc. (London), 1950, A200, 542-554.

37 K. G. Libbrecht, Sol. Phys., 1985, 99, 371-373.

38 M. W. Schmidt and K. Ruedenberg, J. Chem. Phys., 1979, 71, 3951-3962.

39 K. Szalewicz and H. J. Monkhorst, J. Chem. Phys., 1981, 75, $5785-5788$.

40 W. Klopper, J. Chem. Phys., 1995, 102, 6168-6179.

41 D. Bakowies, J. Chem. Phys., 2007, 127, 164109-164120.

42 J. S. Lee and S. Y. Park, J. Chem. Phys., 2000, 112, 10746-10753.

43 C. Schwartz, Phys. Rev., 1962, 128, 1146-1148.

44 F. Jensen, J. Chem. Phys., 1999, 110, 6601-6605.

45 W. Kolos and L. Wolniewicz, J. Chem. Phys., 1965, 43, 2429-2441.

46 S. P. Goldman, Phys. Rev. A: At., Mol., Opt. Phys., 1995, 52, 3718-3729.

47 K. Eichkorn, O. Treutler, H. Öhm, M. Häser and R. Ahlrichs, Chem. Phys. Lett., 1995, 240(4), 283-289.

48 J. Boström, F. Aquilante, T. B. Pedersen and R. Lindh, J. Chem. Theory Comput., 2009, 5(6), 1545-1553.

49 D. E. Knuth, The Art of Computer Programming, Addison-Wesley, Reading, Mass, 1998, vol. 3.

50 P. V. Poblete, J. I. Munro and T. Papadakis, Theor. Comput. Sci., 2006, 352, 136-158 